\title{
ON WAVE PROPAGATION IN LINEAR VISCOELASTICITY*
}

\author{
BY \\ W. J. HRUSA ${ }^{1.2}$ (Carnegie-Mellon University') \\ AND \\ M. RENARDY ${ }^{1.3}$ (Universit! of Wisconsin-Madison)
}

\begin{abstract}
We discuss the initial value problem in one-dimensional linear visco-elasticity with a step-jump in the initial data. If the memory kernel is sufficiently smooth on $[0, \infty)$, the solution exhibits discontinuities propagating along characteristics and a (higher order) stationary discontinuity at the position of the original step-jump. For a singular memory kernel, the propagating waves are smoothed in a manner depending on the nature of the singularity in the kernel, but the stationary discontinuity remains. We also discuss the effects of these phenomena on the regularity of solutions with arbitrary initial data.
\end{abstract}

1. Introduction. In this paper, we study linear wave propagation in a one-dimensional viscoelastic medium. That is, we study the equation

$$
u_{t t}(x, t)=b u_{x x}(x, t)+\int_{-\infty}^{t} m(t-\tau)\left(u_{x x}(x, t)-u_{x x}(x, \tau)\right) d \tau,
$$

$x \in D \subset R, t>0$, where $b$ is a nonnegative constant and $m$ is a positive, monotone decreasing kernel. (For fluids $b=0$, while for solids $b>0$.) We are interested in initial value problems with $D=R$ and discontinuous initial data. More precisely, we shall study situations where $u$ is identically zero for $t<0$, i.e.

$$
u(x, t)=0, \quad x \in R, t<0,
$$

\footnotetext{
* Received July 6, 1984.

'Sponsored by the United States Army under Contract No. DAAG29-80-C-0041.

2This material is based upon work supported by the National Science Foundation under Grant no. MCS-8210950.

${ }^{3}$ This material is based upon work supported by the National Science Foundation under Grant Nos. MCS-8210950 and MCS-8215064.
} 
but there is a spatial jump in the instantaneous values of $u$ and $u_{t}$ at time $t=0$. Specifically, we look at the following types of initial conditions

$$
\begin{array}{ll}
u(x, 0)=\frac{1}{2} \operatorname{sgn} x, \quad u_{t}(x, 0)=0, & x \in R, \\
u(x, 0)=0, \quad u_{t}(x, 0)=\frac{1}{2} \operatorname{sgn} x, & x \in R .
\end{array}
$$

(More general initial conditions can be treated by superposition.)

There have been a number of studies of the so-called Rayleigh problem, i.e. (1.1) on the half line $D=[0, \infty)$ with $u \equiv 0$ for $t<0$ and a step-jump in the boundary value of $u$ at $t=0[2,4-7,11,17,18,20-22,24]$. If the kernel $m$ is sufficiently smooth (including the point 0 ), then the discontinuity at the boundary will propagate with constant speed into the interior, and its amplitude will decay exponentially in time. On the other hand, Renardy [22] has shown that certain singular kernels lead to smoothing of the discontinuity. More precisely, he considered the Rayleigh problem with a class of kernels for which $m(t-\tau)$ behaves like a negative power of $(t-\tau)$ near zero, and showed that that solution is of class $C^{\infty}$ for $t>0$. For cases involving an integrable singularity in $m$, $C^{\infty}$-smoothing coexists with finite speed of propagation [22].

There have been several studies of linear integrodifferential equations in Banach spaces which show that singular kernels can have a regularizing effect on solutions. See, for example, [15] and the references therein.

Hannsgen and Wheeler [14] have shown that the evolution operator associated with (1.1) has a certain compactness property if and only if $m\left(0^{+}\right)=\infty$. This indicated that singular kernels lead to smoothing of solutions, but does not characterize the degree of smoothing. In fact, as we shall see from examples, the degree of smoothing cannot be specified without some definite assumption on the nature of the singularity in $m$.

A new feature that arises in the initial value problem with interior discontinuities in the data is the emergence of stationary singularities in $u$. Initial conditions (1.3a) and (1.3b) lead to solutions with discontinuities in $u_{x x}$ across $x=0$. (A notable exception occurs for $b=0, m(t) \equiv K e^{-\mu t}$, and initial conditions (1.3a), in which case $u$ is smooth across $x=0$ for $t>0$.) The possibility of such stationary singularities for hyperbolic equations with memory was pointed out by Greenberg, Hsiao, and MacCamy [12], [13], [19], but does not seem to have received much attention otherwise. In addition to the stationary singularity, there will be waves propagating to both sides. The propagating waves are given by the same kinds of expressions as those obtained in the Rayleigh problem, and consequently the same regularity results apply.

If $m$ is smooth on $[0, \infty)$, there will be propagating discontinuities. For an appropriate class of kernels with power-type singularities, the propagating waves will be of class $C^{\infty}$. Here we also study kernels with logarithmic singularities, for which we find that the regularity of the propagating waves increases in a manner proportional to time. Sufficiently weak singularities in $m$ (such as log-log) produce only an "infinitesimal" gain in regularity. It is interesting to note that although singular kernels have a smoothing effect on propagating waves, they do not smooth out the stationary discontinuities. 
By superposition, these results can be used to study the regularity of solutions with arbitrary initial data, say of class $H^{s}$. This is discussed briefly in the last section. If $m$ is smooth on $[0, \infty)$, then the strongest singularities in $u$ are always the propagating ones. Roughly speaking, the solution preserves the smoothness of the data. If, however, the kernel has a power-type singularity, the propagating waves will be $C^{\infty}$ and there will be a fixed (but finite) gain in regularity determined by the stationary discontinuities. The case of a logarithmic singularity in $m$ is particularly interesting. The regularity of is determained initially by the propagating waves and increases in a manner proportional to time until the propagating waves and stationary singularities are of the "same strength". Then, the stationary singularities dominate and regularity does not improve any further.

In order to focus ideas and avoid complicated technical hypotheses, we restrict our attention to specific classes of singular kernels that are given by infinite sums of exponentials. (Kernels of a similar structure appear in some rheological models which have been motivated by molecular considerations [9], [23], [25].) It is clear that our arguments can be applied to more general classes of kernels with similar types of singularities.

Throughout this paper, the operations of differentiation, convolution, and Laplace transformation should be interpreted in the sense of tempered distributions. (See, for example, [10].) We shall not dwell on this point. However, we note that the classical and distributional definitions agree for sufficiently regular functions. Moreover, since the distributional definition of an operation typically involves performing the operation on smooth test functions, there is generally no difficulty with changing the order of "limiting" operations. In particular, the order of "differentiation with respect to $x$ " and "Laplace transformation with respect to $t$ " can always be interchanged in the distributional setting.

Acknowledgement. We are indebted to Yuriko Renardy for some valuable suggestions.

2. Initial value problems with step-jump initial conditions. In this section we investigate solutions of (1.1) with $D=R$. We assume throughout that $m$ is nonnegative and nonincreasing, but $m \neq 0$, and that $b \geqslant 0$. We are interested in situations where $u \equiv 0$ for $t<0$, but initial conditions (1.3a) or (1.3b) hold as $t \rightarrow 0^{+}$. To solve (1.1), we introduce Laplace transforms with respect to the time variable:

$$
\hat{u}(x, \lambda)=\int_{0}^{\infty} e^{-\lambda t} u(x, t) d t .
$$

Observe that (1.3a), (1.3b) can be subsumed under

$$
u(x, 0)=\frac{1}{2} \alpha \operatorname{sgn} x, \quad u_{t}(x, 0)=\frac{1}{2} \beta \operatorname{sgn} x .
$$

It is easy to see that $(1.1),(1.2),(2.2)$ lead to the equation

$$
\lambda^{2} \hat{u}(x, \lambda)-\frac{1}{2} \alpha \lambda \operatorname{sgn} x-\frac{1}{2} \beta \operatorname{sgn} x=(b+\hat{m}(0)-\hat{m}(\lambda)) \hat{u}_{x x}(x, \lambda) .
$$

(We remark that " $\hat{m}(0)-\hat{m}(\lambda)$ " should be interpreted as $\int_{0}^{\infty} m(t)\left(1-e^{-\lambda t}\right) d t$, which may converge even if $m$ has a nonintegrable singularity at 0 , i.e. $\hat{m}(0)$ and $\hat{m}(\lambda)$ do not make sense separately.) 
For fixed $\lambda$ this is an ordinary differential equation which is easily solved. We find that

$$
\begin{gathered}
\hat{u}(x, \lambda)=(\alpha \lambda+\beta)\left\{\frac{1}{2 \lambda^{2}} \operatorname{sgn} x-\frac{1}{2 \lambda^{2}} H(x) \exp (-\lambda x / \sqrt{b+\hat{m}(0)-\hat{m}(\lambda)})\right. \\
\left.+\frac{1}{2 \lambda^{2}} H(-x) \exp (\lambda x / \sqrt{b+\hat{m}(0)-\hat{m}(\lambda)})\right\}
\end{gathered}
$$

where $H$ denotes the Heaviside step function. The solution of (1.1), (1.2), (2.2) is obtained by taking the inverse Laplace transform of $\hat{u}$. Although this procedure has been purely formal, it can be justified rigorously under rather mild assumptions on $m$. See [20] for a discussion of this in a similar situation.

For $x, t>0$ we have

$$
u(x, t)=\frac{1}{2}(\alpha+\beta t)-\frac{1}{4 \pi i} \int_{\gamma, i x}^{\gamma+i x}\left(\frac{\alpha}{\lambda}+\frac{\beta}{\lambda^{2}}\right) \exp (\lambda t-\lambda x / \overline{b+\hat{m}(0)} \overline{-\hat{m}(\lambda)}) d \lambda
$$

where $\gamma$ is any positive number, and the integrand is made single-valued in the usual way. A similar expression holds for $x<0$. In fact, $u(-x, t)=-u(x, t)$ for all $x, t>0$. Observe that if $\hat{m}(0)-\hat{m}(\lambda)$ is well defined (in particular, if $m \in L^{1}(0, \infty)$ or if $\int_{0}^{1} t m(t) d t<\infty$ and $\left.\int_{1}^{\infty} m(t) d t<\infty\right)$, then

(i) $\operatorname{Re}(\hat{m}(0)-\hat{m}(\lambda))>0$ for $\operatorname{Re} \lambda>0$;

(ii) $\operatorname{Im}(\hat{m}(0)-\hat{m}(\lambda))>0$ for $\operatorname{Re} \lambda>0, \operatorname{Im} \lambda>0$;

(iii) $\operatorname{Im}(\hat{m}(0)-\hat{m}(\lambda))<0$ for $\operatorname{Re} \lambda>0, \operatorname{Im} \lambda<0$,

by virtue of our sign conditions on $m$.

We note that if $m$ belongs to $L^{1}(0, \infty)$ then the solution of $(1.1),(1.2),(2.2)$ has finite speed of propagation. Indeed, for $m \in L^{1}(0, \infty)$, the Riemann-Lebesgue lemma implies that $\hat{m}(\lambda) \rightarrow 0$ as $\lambda \rightarrow \infty, \operatorname{Re} \lambda \geqslant 0$. Consequently, the contour of integration in (2.5) can be closed by a circle on the right if $x>t, \sqrt{b+\hat{m}(0)}$. Since the integrand has no singularities with $\operatorname{Re} \lambda>0$, it follows from Cauchy's theorem that the integral in (2.5) vanishes for $x>t \cdot \sqrt{b+\hat{m}(0)}$. (See [4].) The situation is analogous for $x<-t$ - $\overline{b+\hat{m}(0)}$.

A. Regular kernels. Our objective here is to describe the regularity of solutions when the kernel $m$ is smooth on $[0, \infty)$. In order to highlight the main ideas and avoid repeated hypotheses, we assume that

$$
m \in C^{\infty}[0, \infty), \quad m^{(k)} \in L^{1}(0, \infty) \quad \forall k=0,1, \ldots
$$

i.e. that $m$ and its derivatives of all ordersr are continuous on $[0, \infty)$ and integrable. It follows that $\hat{m}(\lambda)$ can be approximated asymptotically by a power series in $\frac{1}{\lambda}$ as $\lambda \rightarrow \infty$, $\operatorname{Re} \lambda \geqslant 0$. This can be used to study smoothness properties of the solution. (See [6].)

We first discuss the situation for $x>0$. Away from the line $x=t \cdot \sqrt{b+\hat{m}(0)}, u$ is of class $C^{x}$. Across this line, $u$ sustains a jump discontinuity in case (1.3a), while in case (1.3b), $u$ is continuous but $u_{x}$ and $u_{t}$ jump. The amplitudes of these jumps decay 
exponentially in time. In fact, for $x>0$ the solution of (1.1), (1.2), (2.2) can be written in the form

$$
\begin{aligned}
u(x, t)= & \frac{1}{2}(\alpha+\beta t) \\
& -\frac{1}{2} \alpha \exp \left[-m(0) x / 2(b+\hat{m}(0))^{3 / 2}\right] \cdot H\left(t-\frac{x}{\sqrt{b+\hat{m}(0)}}\right) \\
& -\frac{1}{2}\left[\beta-\frac{m^{\prime}(0) \alpha x}{2(b+\hat{m}(0))^{3 / 2}}-\frac{3 \alpha m(0)^{2} x}{8(b+\hat{m}(0))^{5 / 2}}\right] \exp [-m(0) x / 2 \sqrt{b+\hat{m}(0)}] \\
& \cdot\left(t-\frac{x}{\sqrt{b+\hat{m}(0)}}\right) \cdot H\left(t-\frac{x}{\sqrt{b+\hat{m}(0)}}\right)+F(x, t),
\end{aligned}
$$

where $F$ is of class $C^{1}$ across the line $x=t \cdot \sqrt{b+\hat{m}(0)}$ and of class $C^{x}$ elsewhere, and $F(x, t)=0$ for $x>t \cdot \sqrt{b+\hat{m}(0)}$. Expressions such as (2.7) are, of course, well known. (See, for example, [2].)

The situation is completely analogous for $x<0$. Thus, the behavior of $u$ away from the line $x=0$ is quite similar to the case of a linear wave equation with frictional damping. However, across $x=0$ solutions of (1.1), (1.2), (2.2) generally exhibit discontinuities in $u_{x x}$ which do not occur for equations without memory. (An exception arises in the special case $m(t) \equiv K e^{-\mu t}, b=0$ and initial conditions (1.3a) hold.) Discontinuities of this type have been discussed in a series of papers (concerning Riemann problems for first order hyperbolic equations with memory) by Greenberg, Hsiao, and MacCamy [12], [13], [19], but do not seem to have received much attention otherwise.

Away from the lines $x=0, x= \pm t \cdot \sqrt{b+\hat{m}(0)}, u$ is of class $C^{\infty}$. Moreover, for $t>0$, all derivatives of $u$ have (finite) right and left limits as $x \rightarrow 0$. It is not difficult to show that $u, u_{t}, u_{x}, u_{t t}, u_{t x}$ (and $u_{x t}$ ) are continuous across $x=0$. To investigate the possibility of a stationary discontinuity in $u_{x x}$, we set

$$
J(t)=u_{x x}\left(0^{+}, t\right)-u_{x x}\left(0^{-}, t\right) \quad \forall t>0 .
$$

It follows from (2.4) and a straightforward computation that

$$
\hat{J}(\lambda)=-\frac{\alpha m(0) \hat{m}(\lambda)+\alpha(b+\hat{m}(0))(\lambda \hat{m}(\lambda)-m(0))+\beta(b+\hat{m}(0)) \hat{m}(\lambda)}{(b+\hat{m}(0))^{2}(b+\hat{m}(0)-\hat{m}(\lambda))} .
$$

We note that the distributional derivative $u_{x x}$ is the sum of a function and a distribution supported on the lines $x= \pm t \sqrt{b+\hat{m}(0)}$ (which arises from the propagating jumps described above). The part of $u_{x x}$ which is supported on these lines makes no contribution to $J(t)$ and has been "subtracted off" in our derivation of (2.9). If one were to derive an expression for $\hat{J}(\lambda)$ by simply taking right and left derivatives as $x \rightarrow 0$ in (2.4), then this expression would contain additional terms corresponding to a distribution with support at 0 . 
The first question that we address regarding $J$ is whether or not there are any (nontrivial) situations in which $J$ vanishes identically. It is clear from (2.9) that if $\alpha^{2}+\beta^{2} \neq 0$ and $J \equiv 0$, then $m$ must be an exponential of the form

$$
m(t)=K e^{-\mu t}, \quad K, \mu>0 .
$$

(Recall our sign assumptions on $m$.) Further examination of (2.9) reveals that under initial conditions (1.3a), $J \equiv 0$ if and only if $b=0$ and $m$ is of the form (2.10). Moreover, for initial conditions (1.3b) there are no cases in which $J$ vanishes identically. If values of $\alpha, \beta$ other than those corresponding to (1.3a), (1.3b) are considered, then there will be additional situations in which $J \equiv 0$, e.g. $\alpha=\beta=1, b=\frac{1}{2}, K=1, \mu=2$. (These can easily be characterized using (2.13), (2.14), (2.15) below.)

We note that $J \in C^{\infty}[0, \infty)$. An expression for $J(0)$ can be found by computing $\lim _{\lambda \rightarrow \infty} \lambda \hat{J}(\lambda)$. The outcome of this simple computation is

$$
J(0)=-\frac{\alpha m(0)^{2}+\alpha(b+\hat{m}(0)) m^{\prime}(0)+\beta(b+\hat{m}(0)) m(0)}{(b+\hat{m}(0))^{3}} .
$$

Observe that if $b=0$ and initial conditions (1.3b) hold, then $\hat{J}(\lambda)$ has a pole (or worse) at $\lambda=0$ and consequently one should not expect decay of $J(t)$ as $t \rightarrow \infty$. On the other hand, our sign conditions on $m$ imply that if $b>0$ or if $b=0, \operatorname{tm}(t) \in L^{1}(0, \infty)$, and initial conditions (1.3a) hold, then $\hat{J}(\lambda)$ has no poles with $\operatorname{Re} \lambda \geqslant 0$, and one does expect that $J(t) \rightarrow 0$ as $t \rightarrow \infty$.

By virtue of (2.6), our sign conditions on $m$, and the Riemann-Lebesgue lemma, it follows from (2.9) and Proposition 2.3 of [16] that $J \in L^{1}(0, \infty)$ if $b>0$ or if $b=\beta=0$ and $\operatorname{tm}(t) \in L^{1}(0, \infty)$. By the same kind of reasoning, we find that $J^{\prime}$ also belongs to $L^{1}(0, \infty)$ under either of the above conditions. (In fact, so do all higher order derivatives of $J$.) We therefore conclude that if $b>0$ or if $b=\beta=0$ and $\operatorname{tm}(t) \in L^{1}(0, \infty)$, then $J(t) \rightarrow 0$ as $t \rightarrow \infty$.

A similar argument can be used to handle the case when $b=0$ and initial conditions (1.3b) hold. Examination of (2.8) suggests that $J(t) \rightarrow\left[(\hat{m})^{\prime}(0)\right]^{-1}=-\left(\int_{0}^{\infty} \tau m(\tau) d \tau\right)^{-1}$ as $t \rightarrow \infty$. The idea, then, is to show that $J_{*}, J_{*}^{\prime} \in L^{1}(0, \infty)$, where

$$
J_{*}(t)=J(t)-\left[(\hat{m})^{\prime}(0)\right]^{-1} \quad \forall t \geqslant 0 .
$$

If we assume that $b=0, t^{2} m(t) \in L^{1}(0, \infty)$, and initial conditions (1.3b) hold, then it follows from Proposition 2.3 of [16] and some routine computations that $J_{*}, J_{*}^{\prime} \in L^{1}(0, \infty)$, and consequently $J_{*}(t) \rightarrow 0$ as $t \rightarrow \infty$, i.e. $J(t) \rightarrow-\left(\int_{0}^{\infty} \tau m(\tau) d \tau\right)^{-1}$ as $t \rightarrow \infty$.

The asymptotic behavior of $J$ can thus be summarized as follows. If $b>0$, then $J(t) \rightarrow 0$ as $t \rightarrow \infty$. If $b=0$ and $t^{2} m(t) \in L^{1}(0, \infty)$, then $J(t) \rightarrow-\beta\left(\int_{0}^{\infty} \tau m(\tau) \mathrm{d} \tau\right)^{-1}$ as $t \rightarrow \infty$. This last result suggests that if $b=0$ and $m(t) \sim t^{-(1+\theta)}$ as $t \rightarrow \infty$ with $0<\theta \leqslant 1$, then $J(t) \rightarrow 0$ as $t \rightarrow \infty$; however, we have been unable to prove it. See the paper of Chu [6] for an interesting discussion of the asymptotic behavior of $u$ as $t \rightarrow \infty$ along lines of the form $x=a \cdot t, a>0$.

Since the Laplace transform of a function (or, more generally, a distribution) with compact support is necessarily an entire function, if follows immediately from (2.9) that if 
$b=0$ and $\beta \neq 0$ then the discontinuity in $u_{x x}$ persists for all $t>0$. This is also the case in a number of other situations. In particular, we have been able to show that the support of $J$ is unbounded if $b>0, \alpha \beta \leqslant 0$, and $\alpha^{2}+\beta^{2} \neq 0$, or if $m$ has compact support and $\alpha^{2}+\beta^{2} \neq 0$. The proof is straightforward, but not entirely trivial. We omit the details. (See additional comments concerning completely monotonic kernels in part $\mathrm{E}$ below.)

An interesting expression for $J$ can be obtained by expanding $[b+\hat{m}(0)-\hat{m}(\lambda)]^{-1}$ in powers of $\hat{m}(\lambda) /(b+\hat{m}(0))$ and formally inverting (2.9) as a sum of iterated convolutions. The series for $J$ obtained in this manner converges in $L^{1}(0, \infty)$ if $b>0$ and in $L_{\text {loc }}^{1}[0, \infty)$ if $b=0$. This provides an alternative proof of the integrability of $J$ when $b>0$.

The situation is particularly simple for a single exponential of the form (2.10). As noted above, for $b=0$ and initial conditions (1.3a) we have $J \equiv 0$. Moreover, in this case it is straightforward to show that higher order derivatives of $u$ are continuous across $x=0$. For $b \geqslant 0$ and initial conditions (1.3a), we conclude from (2.8) that

$$
J(t)=\frac{b K \mu}{(b+r)^{3}} \exp [-\mu b t /(b+r)],
$$

where

$$
r=\hat{m}(0)=K / \mu \text {. }
$$

A similar expression holds for initial conditions (1.3b), namely

$$
J(t)=\frac{-K}{(b+r)^{2}} \exp [-\mu b t /(b+r)] .
$$

It is interesting to observe that for $b=0$ and the kernel (2.10), the initial value problem (1.1), (1.2), (2.2) is equivalent to

$$
u_{t t}(x, t)+\mu u_{t}(x, t)=\frac{K}{\mu} u_{x x}(x, t)+\mu \beta \operatorname{sgn} x,
$$

with initial conditions (2.2). We see from Eq. (2.16) that if $\beta \neq 0$ then the solution must have a singularity across $x=0$. Recalling that $u_{t}$ and $u_{t t}$ are continuous across $x=0$ we can deduce directly from (2.16) that $J(t)=-\mu^{2} \beta / K$, which agrees with our previous expressions for this case.

B. Kernels with power-type singularities. In [22], Renardy studied the Rayleigh problem for the class of singular kernels

$$
m(t)=\sum_{n=1}^{\infty} e^{-n^{p} t}, \quad p>\frac{1}{2} .
$$

As $t \rightarrow 0^{+}, m(t)$ behaves like $t^{-1 / p}$. Accordingly, $m$ is integrable if $p>1$. For $p>1$, we have

$$
\hat{m}(\lambda)=\sum_{n=1}^{\infty} \frac{1}{\lambda+n^{p}},
$$

while for $\frac{1}{2}<p \leqslant 1$ we interpret $\hat{m}(0)-\hat{m}(\lambda)$ as

$$
\int_{0}^{\infty}\left(1-e^{-\lambda t}\right) m(t) d t=\sum_{n=1}^{\infty} \frac{\lambda}{n^{p}\left(\lambda+n^{p}\right)} .
$$


Using the results of [22], we can immediately draw the following conclusions regarding (1.1), (1.2), (2.2) with $m$ given by (2.17): For $p>1$ the solution $u$ is analytic away from the lines $x=0, x= \pm t \cdot \sqrt{b+\hat{m}(0)}$, it is of class $C^{x}$ across the lines $x= \pm t \cdot \overline{b+\hat{m}(0)}$, and it propagates with finite speed; for $\frac{1}{2}<p \leqslant 1, u$ is analytic away from the line $x=0$. Thus the singularity in $m$ smooths out the propagating waves. However, it does not smooth out the stationary discontinuity in $u_{x x}$.

By deforming the contour of integration in (2.5) as indicated below, it is straight-forward to show that $u$ and its derivatives of all order have (finite) limits as $x \rightarrow 0^{+}$for $t>0$. For $p>1$, this contour can be used provided that $0<x<t \cdot \sqrt{b+\hat{m}(0)}$; if $\frac{1}{2}<p \leqslant 1$, it can be used for all $x, t>0$. The situation is similar as $x \rightarrow 0^{-}$. Moreover, $u, u_{t}, u_{x}, u_{t t}$, and $u_{t, x}$ are continuous across $x=0$ for $t>0$.

Our previous expression for $\hat{J}(\lambda)$ is not valid under the present circumstances. Nevertheless, it is not too difficult to show that $u_{x x}$ is discontinuous across $x=0$. The argument proceeds roughly as follows. Using (2.4) we find that

$$
\hat{u}_{x x}(x, \lambda) \rightarrow \frac{-(\alpha \lambda+\beta)}{2(b+\hat{m}(0)-\hat{m}(\lambda))} \quad \text { as } x \rightarrow 0^{+} .
$$

(We note that there is no problem with interchanging the order of differentiation with respect to $x$ and taking Laplace transforms-even in the classical sense-for $x>0$.) Suppose that $u_{x x}$ is continuous across $x=0$ for $t>0$. Then, it is easy to see that $u_{x}(0, t)=0$ for all $t>0$ and consequently $u_{x x}(x, t) \rightarrow 0$ uniformly for $t$ in compact subsets of $(0, \infty)$ as $x \rightarrow 0^{+}$. It follows that the expression on the right-hand side of (2.20) must be the Laplace transform of a distribution with support at 0 , i.e. it must be a polynomial in $\lambda$. However, it is easy to show that the expression in question is not a polynomial, and we conclude that $u_{x x}$ is discontinuous across $x=0$ (provided, of course, that $\alpha^{2}+\beta^{2} \neq 0$ ).

The discontinuity in $u_{x x}$ persists for all $t>0$. Indeed, using the contour of Fig. 1 in the inversion integral, one readily sees that the jump $J(t)$ (defined by (2.8)) is analytic in $t>0$. Therefore, since $J \neq 0$, it cannot vanish identically on an interval of the form $[T, \infty)$.

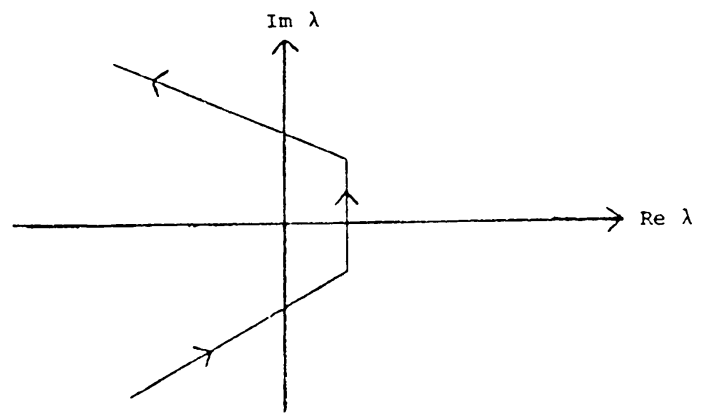

Fig. 1. Contour of Integration. 
One can also argue as follows to show that the support of $J$ is unbounded: If $J$ had compact support then the expression on the right-hand side of (2.20) would be the Laplace transform of a distribution with compact support and hence an entire function of $\lambda$. Examination of (2.18) and (2.19) reveals that this cannot be the case (unless, of course, $\alpha=\beta=0$ ).

C. Kernels with logarithmic singularities. An example of kernel which behaves nicely at infinity and has a logarithmic singularity at zero is given by

$$
m(t)=\sum_{n=0}^{\infty} e^{-e^{n} t} .
$$

To verify this, we note that the asymptotic behavior as $t \rightarrow 0^{+}$does not change if the sum is approximated by an integral. More precisely, using the estimate

$$
\left|\int_{0}^{\infty} f(\nu) d \nu-\sum_{n=1}^{\infty} f(n)\right| \leqslant \int_{0}^{\infty}\left|f^{\prime}(\nu)\right| d \nu
$$

with $f(\nu)=\exp \left(-e^{\nu} t\right)$, we find that

$$
\left|\int_{0}^{\infty} e^{-e^{\nu t}} d \nu-\sum_{n=1}^{\infty} e^{-e^{n} t}\right| \leqslant e^{-t} \quad \forall t>0 .
$$

We then observe that

$$
\begin{aligned}
\int_{0}^{\infty} e^{-e^{\nu} t} d \nu & =\int_{0}^{\infty} e^{-e^{\nu+\ln t} d \nu} \\
& =\int_{\ln t}^{\infty} e^{-e^{\nu}} d \nu=\int_{0}^{\infty} e^{-e^{\nu}} d \nu+\int_{\ln t}^{0} e^{-e^{\mu \nu}} d \nu .
\end{aligned}
$$

The last term in (2.24) clearly has a logarithmic singularity as $t \rightarrow 0^{+}$. We note also that $m \in L^{1}(0, \infty)$.

It follows from (2.21) that

$$
\hat{m}(\lambda)=\sum_{n=1}^{\infty} \frac{1}{\lambda+e^{n}} .
$$

To determine the asymptotic behavior of $\hat{m}(\lambda)$ as $|\lambda| \rightarrow \infty$, we set $\gamma=\operatorname{Re} \lambda, \psi=\operatorname{Im} \lambda$, and observe that

$$
\int_{0}^{\infty} \frac{d \nu}{\lambda+e^{\nu}}=\frac{1}{\lambda} \ln (1+\lambda) .
$$

Using (2.26) and the estimate (2.22) with $f(\nu)=\left(\lambda+e^{\nu}\right)^{-1}$, we deduce that

$$
\hat{m}(\lambda)=\frac{1}{\lambda} \ln (1+\lambda)+O\left(|\psi|^{-1}\right) \quad \text { as }|\psi| \rightarrow \infty .
$$

Therefore, we have

$$
\frac{\lambda}{\sqrt{b+\hat{m}(0)-\hat{m}(\lambda)}}=\frac{\lambda}{(b+\hat{m}(0))^{1 / 2}}+\frac{\ln (1+\lambda)}{2(b+\hat{m}(0))^{3 / 2}}+O(1)
$$


and consequently

$$
\begin{aligned}
& \exp [-\lambda x / \overline{b+\hat{m}(0)-\hat{m}(\lambda)}] \\
&=\exp \left[\frac{-\lambda x}{(b+\hat{m}(0))^{1 / 2}}\right] \cdot \exp \left[\frac{-x \ln (1+\lambda)}{2(b+\hat{m}(0))^{3 / 2}}\right] \cdot \exp [O(1)]
\end{aligned}
$$

uniformly for $x$ in bounded sets as $|\psi| \rightarrow \infty$.

Since $m$ belongs to $L^{1}(0, \infty)$, the integral in (2.5) vanishes if $x>t \cdot \sqrt{b+\hat{m}(0)}$. For $0<x<t \cdot \sqrt{b+\hat{m}(0)}$, we can deform the contour of integration as shown in Fig. 1. Along this contour, the integral is absolutely convergent and remains absolutely convergent if $x$ and $t$ are extended into the complex plane. We can therefore easily conclude that $u$ is analytic in the region $0<x<t \cdot \sqrt{b+\hat{m}(0)}$.

The regularity of $u$ across the line $x=t \cdot \sqrt{b+\hat{m}(0)}$ is directly related to the decay of $\exp \left[-x \ln (1+\lambda) / 2(b+\hat{m}(0))^{3 / 2}\right]$ as $|\lambda| \rightarrow \infty$. Since this term behaves like a negative power of $\lambda$ with exponent proportional to $x$, we can deduce from (2.5), (2.27), and (2.29) that the regularity of $u$ across the line $x=t \cdot \sqrt{b+\hat{m}(0)}$ increases in a manner proportional to $x$ (or equivalently proportional to $t$ ).

It is interesting to observe that discontinuities in the initial data become continuous instantaneously. More precisely, the solution of (1.1), (1.2), (1.3a) is continuous across $x=t \cdot \sqrt{b+\hat{m}(0)}$ for $t>0$, and the solution of (1.1), (1.2), (1.3b) is of class $C^{1}$ across $x=t \cdot \sqrt{b+\hat{m}(0)}$ for $t>0$. This follows immediately from the decay properties of the integrand in (2.5).

The situation is, of course, completely analogous for $x<0$. Arguing as before, one can show that $u$ and its derivatives of all orders have (finite) right and left limits as $x \rightarrow 0$ for $t>0$. Moreover $u, u_{t}, u_{x}, u_{t}$, and $u_{t x}$ are continuous across the line $x=0$ for $t>0$. However, $u_{x x}$ sustains a jump discontinuity across this line; as before the discontinuity persists for all $t>0$ if $\alpha^{2}+\beta^{2} \neq 0$.

D. Kernels with weaker singularities. As the previous examples show, the regularity of $u$ across the lines $x= \pm t \cdot \widehat{b+\hat{m}(0)}$ depends crucially on the behavior of $m(t)$ as $t \rightarrow 0^{+}$. Weak singularities in $m$ generally lead to less smoothing than strong singularities do. In fact, a sufficiently weak singularity in $m$ will produce a gain in regularity which is effectively. infinitesimal. We illustrate this with the kernel

$$
m(t)=\sum_{n=1}^{\infty} \exp \left(-t \cdot e^{e^{n}}\right)
$$

which has a log-log singularity at 0 .

As before, the behavior of $m$ near 0 and the behavior of $\hat{m}$ near infinity to not change if the sum if approximated by an integral. To verify the nature of the singularity in $m$, we observe that for $t<1$,

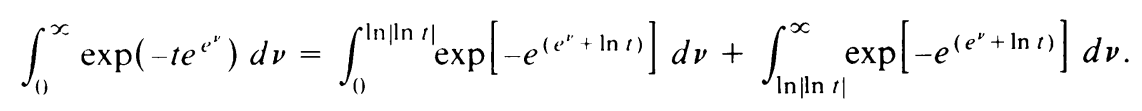


The integrand in the first term on the right-hand side is bounded above and below by positive constants, hence this term behaves like $\ln |\ln t|$ as $t \rightarrow 0^{+}$. With the substitution $\eta=\nu-\ln |\ln t|$, the second integral becomes

$$
\int_{0}^{\infty} \exp \left[-e^{\left(e^{\eta}-1\right) \cdot|\ln t|}\right] d \eta
$$

which is clearly bounded as $t \rightarrow 0^{+}$. Using (2.22), it is easy to see that the difference between $m(t)$ and the integral on the left-hand side of (2.31) remains bounded as $t \rightarrow 0^{+}$. We have thus verified that $m$ has a log-log singularity at 0 .

It follows from (2.30) that

$$
\hat{m}(\lambda)=\sum_{n=1}^{\infty} \frac{1}{\lambda+\exp \left(e^{n}\right)},
$$

and again the asymptotic behavior as $|\lambda| \rightarrow \infty$ does not change if the sum is approximated by an integral. (As in part $C$, the error in this approximation is $O\left(|\psi|^{-1}\right)$.) For real $\lambda$ let us set

$$
I(\lambda)=\int_{0}^{\infty} \frac{d \nu}{\lambda+\exp \left(e^{\nu}\right)} .
$$

With the substitution $w=\exp \left(e^{\nu}\right)$, we find that

$$
\begin{aligned}
I(\lambda) & =\int_{e}^{\infty} \frac{d w}{(\lambda+w) w \ln w} \\
& =\int_{e}^{\lambda} \frac{d w}{(\lambda+w) w \ln w}+\int_{\lambda}^{\infty} \frac{d w}{(\lambda+w) w \ln w} .
\end{aligned}
$$

Expanding $(\lambda+w)^{-1}$ in powers of $w / \lambda$ and $\lambda / w$, respectively, and then making the substitution $v=\ln w$, we see that

$$
\begin{aligned}
I(\lambda) & =\frac{1}{\lambda} \sum_{n=0}^{\infty}(-1)^{n} \lambda^{-n} \int_{e}^{\lambda} \frac{w^{n-1}}{\ln w} d w+\sum_{n=0}^{\infty}(-1)^{n} \lambda^{n} \int_{\lambda}^{\infty} \frac{d w}{w^{n+2} \ln w} \\
& =\frac{1}{\lambda} \ln \ln \lambda+\frac{1}{\lambda} \sum_{n=1}^{\infty}(-1)^{n} \lambda^{-n} \int_{1}^{\ln \lambda} \frac{e^{n !}}{v} d v+\sum_{n=0}^{\infty}(-1)^{n} \lambda^{n} \int_{\ln \lambda}^{\infty} \frac{d v}{v e^{(n+1) k}} \\
= & \frac{1}{\lambda} \ln \ln \lambda+\sum_{n=1}^{\infty} \frac{(-1)^{n}}{\lambda^{n+1}}[\operatorname{Ei}(n \ln \lambda)-\operatorname{Ei}(n)] \\
& +\sum_{n=0}^{\infty}(-1)^{n} \lambda^{n} E_{1}((n+1) \ln \lambda),
\end{aligned}
$$

where Ei denotes the exponential integral. Using formula 5.1.51 on page 231 of [1], we find that, as $\lambda \rightarrow \infty$,

$$
\begin{aligned}
\sum_{n=1}^{\infty} \frac{(-1)^{n}}{\lambda^{n+1}}|E i(n \ln \lambda)-E i(n)| & =\frac{\ln 2}{\lambda \ln \lambda}+O\left(\frac{1}{\lambda(\ln \lambda)^{2}}\right) \\
\sum_{n=0}^{\infty}(-1)^{n} \lambda^{n} E_{1}((n+1) \ln \lambda) & =\frac{\ln 2}{\lambda \ln \lambda}+O\left(\frac{1}{\lambda(\ln \lambda)^{2}}\right) .
\end{aligned}
$$


We therefore conclude that

$$
\int_{0}^{\infty} \frac{d \nu}{\lambda+\exp \left(e^{\nu}\right)}=\frac{1}{\lambda} \ln \ln \lambda+\frac{\ln 2}{\lambda \ln \lambda}+O\left(\frac{1}{\lambda(\ln \lambda)^{2}}\right)
$$

as $\lambda \rightarrow \infty$. By analytic continuation, (2.38) holds for complex $\lambda$ in a right half plane. Since the difference between $\hat{m}(\lambda)$ and the integral in (2.38) is $O\left(|\psi|^{-1}\right)$, we have

$$
\hat{m}(\lambda)=\frac{1}{\lambda} \ln \ln \lambda+O\left(|\psi|^{-1}\right)
$$

as $|\psi| \rightarrow \infty$ for suitable $\gamma>0$. (Recall that $\gamma=\operatorname{Re} \lambda$ and $\psi=\operatorname{Im} \lambda$.)

Away from the lines $x= \pm t \cdot \sqrt{b+\hat{m}(0)}$, the smoothness properties of $u$ are exactly the same as in part $C$. It follows from (2.39) that the integrand in (2.5) can be written in the form

$$
\exp \left[\lambda\left(t-\frac{x}{\sqrt{b+\hat{m}(0)}}\right)\right] \cdot G(x, \lambda) \cdot\left[\frac{\alpha}{\lambda}+\frac{\beta}{\lambda^{2}}\right]
$$

where $G(x, \lambda)$ decays like a negative power of $|\ln \lambda|$ with exponent proportional to $x$. The factor $G(x, \lambda)$ leads to an "infinitesimal" gain in regularity across the line $x=t$ $\cdot \overline{b+\hat{m}(0)}$. The situation is, of course, completely analogous for $x<0$.

It is interesting to observe that for $t$ sufficiently large, the solution of (1.1), (1.2), (1.3a) is continuous across the lines $x= \pm t \cdot \sqrt{b+\hat{m}(0)}$ and the solution of (1.1), (1.2), (1.3b) is of class $C^{1}$ across these lines. (Indeed, the appropriate inversion integrals converge absolutely if $|x|$ is sufficiently large.) We conjecture that the above continuity properties actually hold for all $t>0$, but have been unable to prove it. The difficulty lies in obtaining a suitable error estimate for our approximation of $\hat{m}(\lambda)$. One can show that the principal term in (2.39) leads to a function which is continuous across $x=t \cdot \sqrt{b+\hat{m}(0)}$. However, even though the remainder decays more rapidly than the principal term as $|\lambda| \rightarrow \infty$, its decay rate is not sufficient to guarantee the desired continuity. Thus, more detailed information is needed.

We can prove continuity of the wave front for the kernel given by

$$
m(t)=\int_{0}^{x} \exp \left(-t \cdot e^{e^{x}}\right) d \nu
$$

which also has a log-log singularity at 0 . In this case, we have

$$
\hat{m}(\lambda)=\int_{0}^{\infty} \frac{d \lambda}{\lambda+\exp \left(e^{\nu}\right)},
$$

and consequently, by (2.37)

$$
\hat{m}(\lambda)=\frac{1}{\lambda} \ln \ln \lambda+\frac{\ln 2}{\lambda \ln \lambda}+O\left(\frac{1}{\lambda(\ln \lambda)^{2}}\right)
$$

as $|\psi| \rightarrow \infty$. Once again, it follows that there is only an infinitesimal gain in regularity across the lines $x= \pm t \cdot \sqrt{b+\hat{m}(0)}$. However, the more precise asymptotic estimate (2.43) yields more information concerning continuity. Using (2.43) and formula (9) from 
Section 4.26 of [3], it is not difficult to show that the solution of (1.1), (1.2), (1.3a) is continuous across the lines $x= \pm t \cdot \sqrt{b+\hat{m}(0)}$ for all $t>0$, and the solution of (1.1), (1.2), (1.3b) is of class $C^{1}$ across these lines for all $t>0$.

E. General remarks. It is clear that the above analysis is applicable to more general classes of singular kernels. The crucial factor is the asymptotic behavior of $\hat{m}(\lambda)$ as $\lambda \rightarrow \infty$, which, of course, is related to the behavior of $m(t)$ as $t \rightarrow 0^{+}$. If $m_{1}(t) \sim m_{2}(t)$ as $t \rightarrow 0^{+}$(and some mild regularity conditions hold) then $\hat{m}_{1}(\lambda) \sim \hat{m}_{2}(\lambda)$ as $\lambda \rightarrow \infty$ in any sector of the form $|\arg \lambda| \leqslant \phi<\pi / 2$. (See, for example, Theorem 33.2 of [8].) Unfortunately, such a result is not quite sufficient to give a precise regularity theory for (1.1) which is based solely on the behavior of $m(t)$ as $t \rightarrow 0^{+}$. However, it is easy to see that our arguments can be applied to any kernel whose Laplace transform is suitably behaved in the right half plane.

If $m$ belongs to $L^{1}(0, \infty)$ and is completely monotone (i.e., $m \in C^{\infty}(0, \infty)$ and $(-1)^{k} m^{(k)}(t) \geqslant 0$ for all $\left.t>0, k=0,1, \ldots\right)$, then $\hat{m}$ can be continued analytically to the slit half plane $\mathbb{C} \backslash(-\infty, 0]$ and the contour of Figure 1 can be used in (2.5) for $0<x<t \cdot \sqrt{b+\hat{m}(0)}$. Moreover, $u, u_{t}, u_{x}, u_{t}$, and $u_{t x}$ are continuous across $x=0$ for $t>0$ and the jump $J$ defined by $(2.8)$ is analytic on $(0, \infty)$. The only nontrivial cases in which $J$ vanishes identically occur when $m$ is an exponential of the form (2.10). (See (2.13) and (2.15).) We conjecture that if $m$ is completely monotone, integrable, and $m\left(0^{+}\right)=\infty$, then the solution of (1.1), (1.2), (1.3a) is continuous (and the solution of (1.1), (1.2), (1.3b) is of class $C^{1}$ ) across the lines $x= \pm t \cdot \sqrt{b+\hat{m}(0)}$ for all $t>0$.

It is to be expected that if $m \in C^{\infty}(0, \infty) \cap L^{1}(0, \infty)$, then the solution of (1.1), (1.2), (2.2) is of class $C^{\infty}$ away from the lines $x=0, x= \pm t \cdot \sqrt{b+\hat{m}(0)}$. However, it does not seem easy to prove. A result of this nature is established in the Appendix under an additional assumption on $m$, which, roughly speaking, requires that derivatives of $m$ are not "too wild" near 0 .

3. General initial value problems. Using the results of the preceding section and the method of superposition, it is straightforward to study smoothness properties of solutions to $(1.1),(1.2)$ with initial conditions

$$
u(x, 0)=u_{0}(x), \quad u_{t}(x, 0)=u_{1}(x), \quad x \in R,
$$

for $u_{0}$ and $u_{1}$ in various function classes. Indeed, under rather mild assumptions on $u_{0}$ and $u_{1}$, the solution of (1.1), (1.2), (3.1) can be written in the form

$$
u(x, t)=\int_{-\infty}^{\infty} U_{x}^{a}(x-\xi, t) u_{0}(\xi) d \xi+\int_{-\infty}^{\infty} U_{x}^{h}(x-\xi, t) u_{1}(\xi) d \xi
$$

where $U^{a}$ and $U^{b}$ denote the solutions of (1.1), (1.2), (1.3a) and (1.1), (1.2), (1.3b), respectively. (Recall that derivatives are to be interpreted in the distributional sense.)

We close with an informal discussion of the regularity of solutions of (1.1), (1.2), (3.1). Our assumptions on ( $m$ and $b$ ) are the same as in Sec. 2.

A. Regular kernels. Suppose that $m$ satisfies (2.6). If there is a singularity in the data a point $x_{0} \in R$, then three waves will generally emanate from this point. Singularities will propagate along the lines $x=x_{0} \pm t \cdot \sqrt{b+\hat{m}(0)}$, and (except in very special circumstances) there will be a stationary singularity at $x=x_{0}$ for $t>0$. Such a stationary singularity will always be weaker than the corresponding propagating singularities. 
A discontinuity in $u_{0}^{(k)}$ produces propagating discontinuities in $k$-th derivatives of $u$, while a discontinuity in $u_{1}^{(k)}$ propagates in $(k+1)$-st derivatives of $u$. Discontinuities in $u_{0}^{(k)}$ or $u_{1}^{(k)}$ both lead to stationary discontinuities in $\partial^{k+2} u / \partial x^{k+2}$. If $m$ is a single exponential of the form (2.10), there will be cases in which a singularity in the data does not lead to a stationary singularity in $u$. See Sec. $2 \mathrm{~A}$.

B. Kernels with power type singularities. For the kernel given by (2.17), the propagating waves will always be of class $C^{\infty}$. Consequently, the regularity of $u$ is determined by the stationary singularities. Discontinuities in $u_{0}^{(k)}$ or $u_{1}^{(k)}$ both lead to stationary discontinuities in $\partial^{k+2} u / \partial x^{k+2}$ for $t>0$. Thus, there is a definite (but finite) gain in regularity.

C. Kernels with logarithmic singularities. The situation is especially interesting for the kernel given by (2.21). Initially, the propagating singularities are stronger than the corresponding stationary ones and the regularity of $u$ increases in a manner proportional to time. This will continue until the strongest stationary singularity and the strongest propagating singularity are of the same order. Then, the stationary singularity will dominate and the regularity of $u$ will not improve any further. As before, discontinuities in $u_{0}^{(k)}$ or $u_{1}^{(k)}$ lead to stationary discontinuities in $\partial^{k+2} u / \partial x^{k+2}$.

D. Kernels with weaker singularities. For the kernel (2.30) (or (2.41)), the propagating singularities are stronger than the corresponding stationary ones for all time. The smoothing of the propagating waves leads to only an infinitesimal gain in regularity of $u$.

For the kernel (2.41), we can, however, make a definite statement concerning continuity. If $u_{0} \in \mathrm{BV}(\mathbb{R})$ and $u_{1} \equiv 0$, the solution of (1.1), (1.2), (3.1) is continuous on $R \times(0, \infty)$; if $u_{0} \equiv 0$ and $u_{1} \in \mathrm{BV}(R)$, then the solution of (1.1), (1.2), (3.1) is of class $C^{1}$ on $R \times(0, \infty)$. We conjecture that this is also true for the kernel $(2.30)$ (and, more generally for any completely monotonic $m \in L^{1}(0, \infty)$ ).

Appendix. In this appendix, we discuss regularity of the solution $u$ of (1.1), (1.2), (2.2) away from the lines $x=0, x= \pm t \cdot \sqrt{b+\hat{m}(0)}$, under the assumptions that $m$ is nonnegative, nonincreasing, and

$$
m \in C^{\infty}(0, \infty) \cap L^{1}(0, \infty)
$$

and that $b \geqslant 0$. From previous considerations, we know that the integral in (2.5) vanishes for $x>t \cdot \sqrt{b+\hat{m}(0)}$. Moreover, $u(-x, t)=-u(x, t)$ for all $x, t>0$. It therefore suffices to study the case $0<x<t \cdot \sqrt{b+\hat{m}(0)}$. For convenience, we set

$$
B=b+\hat{m}(0) \text {. }
$$

Proposition. Assume that $b \geqslant 0, m$ is nonnegative and nonincreasing, and (a.1) holds. Assume further that for each $k=0,1, \ldots$, there exists a number $N_{k}>0$ such that

$$
\limsup _{t \rightarrow 0^{+}} t^{N_{k}}\left|m^{(k)}(t)\right|<\infty \text {. }
$$

Then, for each $x>0$, the solution $u$ of (1.1), (1.2), (2.2) is a $C^{\infty}$ function of $t$ for $t>x / \sqrt{B}$, i.e. $u(x, \cdot) \in C^{\infty}(x / \sqrt{B}, \infty)$.

REMARK. An expanded version of the proof outlined below can be used to show that $u$ is (jointly) of class $C^{\infty}$ on the domain $\{(x, t): 0<x<t \cdot \sqrt{B}\}$. 
Sketch of proof. The argument will be partitioned into several steps. Since we are interested only in smoothness (and not in asymptotic behavior as $t \rightarrow \infty$ ), we assume, without loss of generality, that the support of $m$ is bounded.

Step 1. Consider the function $f$ whose Laplace transform is given by

$$
\hat{f}(\lambda)=\sqrt{B-\hat{m}(\lambda)}-\sqrt{B} .
$$

By virtue of (a.1), our sign conditions on $m$ and $b$, and the Riemann-Lebesgue lemma, it follows from Proposition 2.3 of [16] that $\hat{f}$ is the Laplace transform of a function $f \in L^{1}(0, \infty)$. We want to show that, in addition,

$$
f \in C^{\infty}(0, \infty) \text {. }
$$

Observe that $f$ satisfies the equation

$$
(f+\sqrt{B} \delta) *(f+\sqrt{B} \delta)=B \delta-m,
$$

or

$$
(f * f)(t)+2 \sqrt{B} f(t)=-m(t)
$$

where $\delta$ is the Dirac distribution and the $*$ denotes convolution on $[0, t]$. We can always write $m$ in the form $m=m_{1}+m_{2}$, where

$$
\hat{m}_{1}(\lambda)=O\left(\frac{1}{\lambda^{n}}\right) \text { as } \lambda \rightarrow \infty, \operatorname{Re} \lambda \geqslant 0
$$

for every $n>0$ and $m_{2}$ is nonnegative, nonincreasing, and satisfies

$$
m_{2} \in L^{1}(0, \infty)
$$

with $\left\|m_{2}\right\|_{L^{1}}$ arbitrarily small. (Note that $t m_{2}^{\prime}(t) \in L^{1}(0, \infty)$ and $\left\|t m_{2}^{\prime}(t)\right\|_{L^{1}}=\left\|m_{2}\right\|_{L^{1}}$.)

It follows from (a.7) that $m_{1} \in C^{\infty}[0, \infty)$, and consequently derivatives of $m_{2}$ can grow no faster than derivatives of $m$ near 0 . It also follows that

$$
\sqrt{B-\hat{m}(\lambda)}-\sqrt{B-\hat{m}_{2}(\lambda)}=0\left(\frac{1}{\lambda^{n}}\right)
$$

for every $n>0$ as $\lambda \rightarrow \infty$. Therefore, to discuss the regularity of $f$, it suffices to consider

$$
f_{2} * f_{2}+2 \sqrt{B} f_{2}=-m_{2} \text {. }
$$

By choosing $m_{2}$ sufficiently small, we can guarantee that (a.11) has a solution $f_{2} \in L^{1}(0, \infty)$ with $\left\|f_{2}\right\|_{L^{1}}$ arbitrarily small.

We rewrite (a.11) as

$$
2 \sqrt{B} f_{2}(t)+2 \int_{0}^{t / 2} f_{2}(t-\tau) f_{2}(\tau) d \tau=-m_{2}(t)
$$

from which we find formally

$$
2 \sqrt{B}\left\|f_{2}\right\|_{L^{\infty}[t, \infty)}-2\left\|f_{2}\right\|_{L^{\infty}[t / 2, \infty)} \cdot\left\|f_{2}\right\|_{L^{1}(0, \infty)} \leqslant\left\|m_{2}\right\|_{L^{\infty}[t, \infty)} .
$$

For each $n=1,2, \ldots$, let us set

$$
\begin{aligned}
M_{n} & =K^{-n}\left\|m_{2}\right\|_{L^{\infty}\left[2^{-n}, \infty\right)}, \\
F_{n} & =K^{-n}\left\|f_{2}\right\|_{L^{\infty}\left[2^{-n}, \infty\right)},
\end{aligned}
$$


where $K$ is a suitable positive constant (to be chosen later). It follows from (a.13) that

$$
2 \sqrt{B} \sum_{n=1}^{\infty} F_{n}-2 K\left\|m_{2}\right\|_{L^{1}} \sum_{n=1}^{\infty} F_{n+1} \leqslant \sum_{n=1}^{\infty} M_{n} .
$$

If $K$ is large enough, then $\sum_{n=1}^{\infty} M_{n}<\infty$, and (a.16) yields a bound for $\sum_{n=1}^{\infty} F_{n}$ provided that $K\left\|m_{2}\right\|_{I^{1}}$ is sufficiently small. By formally differentiating (a.12), we get the same kind of estimates for derivatives of $m_{2}$ by virtue of (a.3).

We can now prove that $f \in C^{\infty}(0, \infty)$ as follows. Let $N$ be a fixed (but arbitrary) positive integer. Then, we write $m=m_{1}+m_{2}$, with $m_{1}$ and $m_{2}$ as above and $m_{2}$ small enough so that the procedure outlined above yields estimates for derivatives of $m_{2}$ through $\operatorname{order} N$. For each $\varepsilon>0$, we set

$$
m_{2,}(t)=m_{2}(t+\varepsilon), \quad t \geqslant 0,
$$

and denote by $f_{2}$, the solution of

$$
2 \sqrt{B} f_{2,}(t)+2 \int_{0}^{t / 2} f_{2,}(t-\tau) f_{2,}(\tau) d \tau=-m_{2,}(t)
$$

It is clear that $f_{2} \in C^{\infty}[0, \infty)$ for each $\varepsilon>0$. Moreover, our estimates for derivatives of $m_{2}$ hold for derivatives of $m_{2,}$-independently of $\varepsilon$. As $\varepsilon \rightarrow 0, f_{2} \rightarrow f_{2}$ in $L^{1}(0, \infty)$. In addition, $f_{2}$, and its derivatives through order $N$ are uniformly bounded on compact subsets of $(0, \infty)$, and consequently $f_{2} \in C^{N-1}(0, \infty)$. Since $f-f_{2} \in C^{\infty}[0, \infty)$, we have $f \in C^{N-1}(0, \infty)$. Finally, since $N$ was arbitrary, we conclude that $f \in C^{\infty}(0, \infty)$.

This procedure also shows that derivatives of $f_{2}$ satisfy bounds of the form (a.3), independently of the particular decomposition of $m$ into $m_{1}+m_{2}$. Moreover, $t f_{2}^{\prime}(t) \in$ $L^{1}(0, \infty)$ with $\left\|t f_{2}^{\prime}(t)\right\|_{L^{1}}$ arbitrarily small if $m_{2}$ is sufficiently small.

Step 2. Next, consider the function $g$ whose Laplace transform is given by

$$
\hat{g}(\lambda)=\frac{1}{\sqrt{B}}-\frac{1}{\sqrt{B-\hat{m}(\lambda)}}=\frac{1}{\sqrt{B}}-\frac{1}{\hat{f}(\lambda)+\sqrt{B}} .
$$

As in Step 1, it follows from Proposition 2.3 of [16] that $\hat{g}$ is the Laplace transform of a function $g \in L^{1}(0, \infty)$. We note that $g$ satisfies

$$
\left(\frac{\delta}{\sqrt{B}}-g\right) *(\sqrt{B} \delta+f)=\delta,
$$

or

$$
\sqrt{B} g+f * g=\frac{f}{\sqrt{B}} .
$$

As before, to study the smoothness properties of $g$, it is enough to consider

$$
\sqrt{B} g_{2}+f_{2} * g_{2}=\frac{1}{\sqrt{B}} f_{2} \text {. }
$$

A modified version of the argument in Step 1 shows that $g$ and $g_{2}$ have the same regularity properties as $f$ and $f_{2}$, respectively. Moreover, $\left\|g_{2}\right\|_{L^{1}}$ and $\left\|\operatorname{tg}_{2}^{\prime}(t)\right\|_{L^{1}}$ can be made arbitrarily small by choosing $m_{2}$ sufficiently small. 
Step 3. Let $c$ be a positive constant and consider the function $h$ whose Laplace transform is given by

$$
\hat{h}(\lambda)=\frac{1}{\lambda} \exp (c \lambda \hat{g}(\lambda)) \text {. }
$$

From sign conditions on the kernel, etc., we know that $h \in L_{\text {loc }}^{2}[0, \infty)$. Observe that $\hat{h}$ satisfies the differential equation

$$
\frac{d}{d \lambda} \hat{h}(\lambda)=-\frac{1}{\lambda} \hat{h}(\lambda)+\frac{d}{d \lambda}(c \lambda \hat{g}(\lambda)) \cdot \hat{h}(\lambda),
$$

which translates into

$$
\operatorname{th}(t)=\int_{0}^{t} h(\tau) d \tau+c \int_{0}^{t} \tau g^{\prime}(\tau) h(t-\tau) d \tau
$$

As in Step 1, to study the smoothness properties of $h$, it is enough to consider the part of $h$ which corresponds to $m_{2}$, i.e. the solution $h_{2}$ of

$$
t h_{2}(t)=\int_{0}^{t} h_{2}(\tau) d \tau+c \int_{0}^{t} \tau g_{2}^{\prime}(\tau) h_{2}(t-\tau) d \tau .
$$

Indeed, it is easy to see that $h-h_{2} \in C^{\infty}[0, \infty)$. The argument of Step 1 cannot be applied directly to (a.26) because of the singular coefficient on the left-hand side. To overcome this difficulty, we fix $t_{0}>0$ and set

$$
\phi_{t_{0}}(t)=h_{2}\left(t+t_{0}\right), \quad t \geqslant 0 .
$$

After several changes of variable, (a.26) yields

$$
\begin{aligned}
\left(t+t_{0}\right) \phi_{t_{0}}(t)= & \int_{0}^{t} \phi_{t_{0}}(\tau) d \tau+c \int_{0}^{t / 2} \tau g_{2}^{\prime}(\tau) \phi_{t_{0}}(t-\tau) d \tau \\
& +c \int_{t / 2}^{t} \tau g_{2}^{\prime}(\tau) \phi_{t_{0}}(t-\tau) d \tau+\int_{0}^{t_{0}} h_{2}(\tau) d \tau \\
& +c \int_{0}^{t_{0}}\left(t+t_{0}-\tau\right) g_{2}^{\prime}\left(t+t_{0}-\tau\right) h_{2}(\tau) d \tau
\end{aligned}
$$

We know a priori that $\phi_{t_{0}}, h_{2} \in L_{\text {loc }}^{2}[0, \infty)$. Moreover, we have information on $g_{2}$ from Step 2. A modified version of the procedure of Step 1 can now be used to show that $h \in C^{\infty}(0, \infty)$. (In this case intervals of the form $\left[2^{-n}, T\right]$ with $T<\infty$ should be used in place of $\left[2^{-n}, \infty\right)$.)

Step 4. For $\alpha=1, \beta=0$, the integrand in (2.5) can be written in the form

$$
\exp \left[\lambda\left(t-\frac{x}{\sqrt{B}}\right)\right] \cdot \frac{1}{\lambda} \cdot \exp (x \lambda \hat{g}(\lambda)) .
$$

(The case of general $\alpha, \beta$ follows easily from this one.) We can therefore conclude from Step 3 that for each (fixed) $x>0, u$ is a $C^{\infty}$ function of $t-x / \sqrt{B}$ for $t-x / \sqrt{B}>0$, and consequently $u(x, \cdot) \in C^{\infty}(x / \sqrt{B}, \infty)$.

\section{REFERENCES}

[1] M. Abramowitz and I. A. Stegun, Handhook of mathematical functions, Dover, 1965

[2] J. D. Achenbach and D. P. Reddy, Note on wave propagation in linearly viscoelastic media, Z. Angew. Math. Phys. 18, 141-144 (1967)

[3] Bateman Project, Tables of integral transforms, Vol. 1, McGraw-Hill, 1954, p. 225 
[4] D. S. Berry, A note on stress pulses in viscoelastic rods, Phil. Mag., Ser. 8, 100-102 (1958)

[5] R. M. Christensen, Theory of viscoelasticity, Academic Press, 1971

[6] B. T. Chu, Stress waves in isotropic linear viscoelastic materials, J. Mécanique 1, 439-446 (1962)

[7] B. D. Coleman, M. E. Gurtin and I. R. Herrera, Waves in materials with memory, Arch. Rational Mech. Anal. 19. 1-19 and 239-265 (1965)

[8] G. Doetsch, Introduction to the theory and application of the Laplace transformation, Springer, 1974

[9] M. Doi and S. F. Edwards, Dynamics of concentrated polymer systems, J. Chem. Soc. Faraday 74, 1789-1832 (1978) and 38-54, 75, (1979)

[10] W. F. Donoghue, Distributions and Fourier transforms, Academic Press, 1969

[11] G. M. C. Fisher and M. E. Gurtin, Wave propagation in the linear theory of viscoelasticity, Q. Appl. Math. 23. $257-263(1965)$

[12] J. M. Greenberg. L. Hsiao and R. C. MacCamy, A model Riemann problem for Volterra equations, in Volterra and Functional Differential Equations, K. B. Hannsgen et al. (ed.), Dekker, 25-43, 1982

[13] J. M. Greenberg and L. Hsiao, The Riemann problem for the system $u_{t}+\sigma_{\mathrm{x}}=0$ and $(\sigma-f(u))_{t}+(\sigma-$ $\mu f(u))=0$, Arch. Rational Mech. Anal. 82, 87-108 (1983)

[14] K. B. Hannsgen and R. L. Wheeler, Behavior of the solutions of a Volterra equation as a parameter tends to infinity, J. Integral Equations (to appear)

[15] M. Ianelli, Some results on linear integro-differential equations in a Banach space (preprint)

[16] G. S. Jordan, O. J. Staffans and R. L. Wheeler, Local analyticity in weighted $L^{1}$-spaces and applications to stability problems for Volterra equations, Trans. Amer. Math. Soc. 174, 749-782 (1982)

[17] J. Y. Kazakia and R. S. Rivlin, Run-up and spin-up in a viscoelastic fluid, Rheol. Acta 20, 111-127 (1981)

[18] E. H. Lee and J. A. Morrison, A comparison of the propagation of longitudinal waves in rods of viscoelastic materials, J. Polymer Sci. 19, 93-110 (1956)

[19] R. C. MacCamy, A model Riemann problem for Volterra equations, Arch. Rational Mech. Anal. 82, 71-86 (1983)

[20] A. Narain and D. D. Joseph, Linearized dynamics for step jumps of velocity and displacement of shearing flows of a simple fluid, Rheol. Acta 21, 228-250 (1982)

[21] A. C. Pipkin, Lectures on viscoelasticity theory, Springer, 1972

[22] M. Renardy, Some remarks on the propagation and non-propagation of discontinuities in linearly viscoelastic liquids, Rheol. Acta 21, 251-254 (1982)

[23] P. E. Rouse, A theory of the linear viscoelastic properties of dilute solutions of coiling polymers, J. Chem. Phys. 21, 1271-1280 (1953)

[24] R. I. Tanner, Note on the Rayleigh problem for a viscoelastic fluid, Z. Angew. Math. Phys. 13, 573-580 (1962)

[25] B. H. Zimm, Dynamics of polymer molecules in dilute solutions: viscoelasticity, flow birefringence and dielectric loss, J. Chem. Phys. 24, 269-278 (1956) 